\title{
Imitators And Optimizers in COURnot Oligopoly*
}

\author{
Burkhard C. Schipper ${ }^{\dagger}$ \\ Department of Economics \\ University of California, Davis
}

February 11, 2008

\begin{abstract}
We analyze a symmetric n-firm Cournot oligopoly with a heterogeneous population of optimizers and imitators. Imitators mimic the output decision of the most successful firms of the previous round a là Vega-Redondo (1997). Optimizers play a myopic best response to the opponents' previous output. Firms make mistakes and deviate from their decision rules with a small probability. Applying stochastic stability analysis, we find that the long run distribution converges to a recurrent set of states in which imitators are better off than are optimizers.
\end{abstract}

Keywords: profit maximization hypothesis, bounded rationality, learning, Stackelberg, quasisubmodularity.

JEL-Classifications: C72, D21, D43, L13.

*I am grateful to the editor, Cars Hommes, and three referees for helpful comments. Moreover, I thank Carlos Alós-Ferrer, Itzhak Gilboa, Jörg Oechssler, and Yossi Spiegel as well as audiences of various seminars and conferences for helpful discussions. A first version of this paper was presented at WEHIA Maastricht 2001. Financial support from the Deutsche Forschungsgemeinschaft through SFB/TR 15 and the DAAD is gratefully acknowledged.

${ }^{\dagger}$ Department of Economics, University of California, Davis, One Shields Avenue, Davis, CA 95616, USA, Phone: +1-530-752 6142, Fax: +1-530-752 9382, Email: bcschipper@ucdavis.com 
"Men nearly always follow the tracks made by others and proceed in their affairs by imitation, even though they cannot entirely keep to the tracks of others or emulate the prowess of their models. So a prudent man should always follow in the footsteps of great men and imitate those who have been outstanding." Niccolò Machiavelli

\section{Introduction}

One of the most fundamental assumptions in economics is that firms maximize absolute profits. However, already Alchian (1950) suggested that firms may maximize relative profits in the long run rather than absolute profits. In contrast, Friedman (1953) argued that evolutionary selection forces favor absolute profit maximization. In particular, he postulated that, although firms may not know their profit functions, we can assume that they behave as if they maximize profits because otherwise they would be driven out of the market by firms that do behave as if they maximize profits. Koopmans (1957), p. 140, remarked that if selection does lead to profit maximization then such an evolutionary process should be part of economic modeling. Following Koopmans' suggestion, this paper shows that there is an interesting class of counterexamples to Friedman's conjecture.

The present paper was inspired by Vega-Redondo (1997). ${ }^{1}$ He shows that in a quantity setting symmetric n-firm Cournot oligopoly with imitators, the long run outcome converges to the competitive output if small mistakes are allowed. Imitators mimic the output of the most successful firms in the previous round. His result is in sharp contrast to optimizers, whose outputs are known to converge under certain conditions in the Cournot tatonnement to the Cournot Nash equilibrium. It seems natural to wonder what happens if imitators and optimizers are mixed together in a heterogeneous population. According to Friedman, we should find that optimizers are better off than are imitators, and that consequently optimizers drive out imitators in any payoff monotone selection dynamics. However, we find that imitators are strictly better off than are optimizers, which is at first glance a rather surprising result given that imitators are less sophisticated than optimizers. In a sense, this result is reminiscent of Stackelberg behavior. That's why we name the support of the long run distribution the set of Pseudo-Stackelberg

\footnotetext{
${ }^{1}$ See also Schaffer (1989), Rhode and Stegeman (2001), Alós-Ferrer (2004) and Thijssen (2005).
} 
states. Imitators and optimizers play roles analogous to those of the "independent" and the "dependent" firms respectively in von Stackelberg's (1934) work. ${ }^{2}$ Optimizers are "dependent" since by definition they play a best response. Imitators are "independent" because they do not perceive any influence on the price. Analogous to the profits of von Stackelberg's independent and dependent firms, every imitator is better off than every optimizer.

In the proofs of our results, we rely on the quasisubmodularity of payoff functions (see Topkis, 1998, pp. 43, Milgrom and Shannon, 1994). The intuition for quasisubmodularity in our context is that if a firm prefers a larger quantity to a lower quantity for a given total market quantity, then it prefers also the larger quantity to the lower quantity for a lower total market quantity. The Cournot oligopoly satisfies this property by definition (see Lemma 1). A similar version of this property, in which the total market quantity is replaced by the opponents' quantity, is used in modern oligopoly theory (e.g. Vives 2000, Amir, 1996, Amir and Lambson, 2000). Vega-Redondo's (1997) result has been generalized to the class of aggregative quasisubmodular games (see Schipper, 2003). The results in this paper are likely to generalize as well to aggregative quasisubmodular games such as some rent seeking games, some common pool resource games etc. For instance, consider a repeated Nash demand game and suppose that the imitator demands a share larger than $50 \%$ of the pie. What can an optimizer do? It can optimize by demanding the highest share compatible to the claim of the imitator. If the optimizer demands less then it forgoes profits. If the optimizer demands more then both make zero profits. Assuming that the imitator mimics itself in such situation we can conclude that the optimizer can not manipulate the decision of the imitator in its favor. Hence it appears that also in this repeated Nash demand game the imitator is better off than is the optimizer.

The dynamic analysis in this paper uses the concept of stochastic stability. The general idea is that mutations select among absorbing sets of the decision process such that only the most robust absorbing sets remain in the support of the limiting invariant distribution. We interpret these mutations as bounded rationality of the firms. That is, there is always a small positive probability of making mistakes in output decisions. A necessary condition for a state to be contained in the support of the unique invariant limiting distribution is that this state is contained in the minimal set of absorbing sets that is robust to a single mutation. Such

\footnotetext{
${ }^{2}$ It is interesting to note that von Stackelberg himself never used the word "leader" in his book but spoke of the "independent" and the "dependent" firm.
} 
a set is called a recurrent set (see Samuelson, 1997). In our main result we show that the symmetric Cournot Nash equilibrium, the only absorbing state in which optimizers are as well off as imitators, is not the unique stochastically stable state. Moreover, we also show in an example that there are parameters of the game such that the entire set of Pseudo-Stackelberg states is the unique recurrent set. In any case, the support of the unique limiting invariant distribution implies that imitators are strictly better off than are optimizers.

The paper is organized as follows: Section 2 introduces the model and the decision rules. It is followed in section 3 by a discussion of candidates for solutions. Section 4 presents the results, which are subsequently discussed in the concluding section 5. All proofs are contained in the appendix.

\section{Model}

Consider a symmetric Cournot model with $N=\{1,2, \ldots, n\}$ firms that produce a homogeneous good. The cost function of each firm $i$ is $c\left(q_{i}\right)$, where $q_{i} \in \mathbb{R}$ is the output of firm $i$ and the inverse demand function is $p(Q)$, where $Q:=\sum_{i \in N} q_{i}$ is the total output over all firms. It will be convenient to write profits as a function of the individual quantity and the total quantity,

$$
\pi_{i}\left(q_{i}, Q\right):=q_{i} p(Q)-c\left(q_{i}\right), \text { for all } i \in N
$$

We restrict our analysis to a symmetric oligopoly since imitation is more reasonable if firms face similar conditions of production. ${ }^{3}$

We assume that firms choose output from a common finite grid $\Gamma=\{0, \delta, 2 \delta, \ldots, \nu \delta\}$, where both $\delta>0$ and $\nu \in \mathbb{N}$ are arbitrary. This turns the strategic situation into a finite game and allows us to focus on finite Markov chains later in the dynamic analysis.

Our proofs rely heavily on the following property:

Definition 1 (Quasisubmodularity) $\pi_{i}$ is strictly quasisubmodular in $\left(q_{i}, Q\right)$ on $\Gamma \times\{0, \delta, 2 \delta, \ldots, n \nu \delta\}$ if for all $q_{i}^{\prime \prime}>q_{i}^{\prime}, Q^{\prime \prime}>Q^{\prime}$

$$
\pi_{i}\left(q_{i}^{\prime \prime}, Q^{\prime \prime}\right) \geq \pi_{i}\left(q_{i}^{\prime}, Q^{\prime \prime}\right) \Longrightarrow \pi_{i}\left(q_{i}^{\prime \prime}, Q^{\prime}\right)>\pi_{i}\left(q_{i}^{\prime}, Q^{\prime}\right)
$$

\footnotetext{
${ }^{3}$ Our model could be extended to asymmetric settings, in which each firm may imitate opponents that are most similar to her in terms of the payoff function.
} 


$$
\pi_{i}\left(q_{i}^{\prime}, Q^{\prime}\right) \geq \pi_{i}\left(q_{i}^{\prime \prime}, Q^{\prime}\right) \Longrightarrow \pi_{i}\left(q_{i}^{\prime}, Q^{\prime \prime}\right)>\pi_{i}\left(q_{i}^{\prime \prime}, Q^{\prime \prime}\right)
$$

Assumption 1 (Strictly Decreasing Demand) For all $Q, Q^{\prime} \in\{0, \delta, 2 \delta, \ldots, n \nu \delta\}$, if $Q^{\prime}>$ $Q$ then $p\left(Q^{\prime}\right)<p(Q)$.

Lemma 1 If Assumption 1 holds, then for any $i \in N, \pi_{i}$ is strictly quasisubmodular in $\left(q_{i}, Q\right)$ on $\Gamma \times\{0, \delta, 2 \delta, \ldots, n \nu \delta\}$.

The property allows us to compare profits of firms operating with different quantities in the same market. Note that this property follows directly from the structure of the Cournot game. No additional assumptions on costs have to be imposed.

The dynamics of the system is assumed to proceed in discrete time, $t=0,1,2, \ldots$. At each $t$ the state of the system is identified by the current output schedule $\omega(t)=\left(q_{1}(t), q_{2}(t), \ldots, q_{n}(t)\right)$. The state space of the system is identical to $\Gamma^{n}$. Associated with any such state $\omega(t) \in \Gamma^{n}$ is the induced profit profile $\pi(t)=\left(\pi_{1}(t), \pi_{2}(t), \ldots, \pi_{n}(t)\right)$ at $t$, defined by $\pi_{i}(t):=q_{i}(t) p(Q(t))-$ $c\left(q_{i}(t)\right)$, for all $i \in N$.

At every time $t=1,2, \ldots$, each firm $i \in N$ has regardless of history an i.i.d. probability of being able to revise her former output $q_{i}(t-1)$. We assume that this probability is bounded away from zero and one, hence the process has inertia. That is, not every period all firms adjust their output. The idea is that it may be too costly to always adjust output.

The finite population of firms $N$ is partitioned into two subpopulations of imitators and optimizers respectively. Let $I$ be the subset of $N$ that contains all imitators. The fraction of imitators in the population is denoted by $\theta=\frac{\sharp I}{\sharp N}$. The firms in the two subpopulations are characterized by different decision rules.

Definition 2 (Imitator) An imitator $i \in I$ chooses with full support from the set

$$
D_{I}(t-1):=\left\{q \in \Gamma: \exists j \in N \text { s.t. } q=q_{j}(t-1) \text { and } \forall k \in N, \pi_{j}(t-1) \geq \pi_{k}(t-1)\right\} \text {. }
$$

That is, every period there exists a firm $j$ that had the highest profit in the previous period. An imitator imitates the previous period's quantity of firm $j$. It is the same imitation rule as in Vega-Redondo (1997). 
Definition 3 (Optimizer) An optimizer $i \in N \backslash I$ chooses from the set

$$
D_{O}(t-1):=\left\{q \in \Gamma: q \in b\left(q_{-i}(t-1)\right)\right\}
$$

with $q_{-i}:=\sum_{j \in N \backslash\{i\}} q_{j}$ and $b:\{0, \delta, 2 \delta, \ldots,(n-1) \nu \delta\} \longrightarrow \Gamma$ is the firm's best response correspondence defined by

$$
b\left(q_{-i}\right):=\left\{q_{i}^{\prime} \in \Gamma: q_{i}^{\prime} p\left(q_{-i}+q_{i}^{\prime}\right)-c\left(q_{i}^{\prime}\right) \geq q_{i} p\left(q_{-i}+q_{i}\right)-c\left(q_{i}\right), \forall q_{i} \in \Gamma\right\} .
$$

Definition 3 means that an optimizer sets an output level that is a best response to the opponents' total output in the previous period.

It is assumed that initially in $t=0$ every firm starts with an arbitrary output in $\Gamma$. The process induced by the decision rules is a $n$-vector discrete time finite Markov chain with stationary transition probabilities.

At every output revision opportunity $t$, each firm follows her decision rule with probability $(1-\varepsilon), \varepsilon \in(0, a], a<1$, and with probability $\varepsilon$ she randomizes with full support $\Gamma$. As a matter of convention, we call a firm mutating at $t$ if she randomizes with full support at $t$. The noise has a convenient technical property: Let $P(\varepsilon)$ be the Markov matrix $P$ perturbed with the level of noise $\varepsilon . P(\varepsilon)$ is regularly perturbed (Young, 1993, p. 70), i.e., it is an ergodic and irreducible Markov chain on $\Gamma^{n}$. This implies that there exists a unique invariant distribution $\varphi(\varepsilon)$ on $\Gamma^{n}$ (for standard results on Markov processes see for example Masaaki, 1997). To put it more intuitively, the noise makes any state accessible from any other state in finite time. This is sufficient for the existence of the unique invariant distribution.

The following analysis focuses on the unique limiting invariant distribution $\varphi^{*}$ of $P$ defined by $\varphi(\varepsilon) P(\varepsilon)=\varphi(\varepsilon), \varphi^{*}:=\lim _{\varepsilon \rightarrow 0} \varphi(\varepsilon)$ and $\varphi^{*} P=\varphi^{*}$. In particular, the focus is on how to characterize this probability vector since it provides a description of the long run output behavior of the market when the noise goes to zero. This long run distribution describes the average proportion of time spent in each state in the long run, or expressed differently, the relative frequency of a state's appearance as the time goes to infinity (see Samuelson, 1997). 


\section{Candidates for Solutions}

By standard results (e.g. see Samuelson, 1997, Proposition 7.4) we know that the support of the long run distribution can only contain states that are elements of absorbing sets of the unperturbed process. Therefore we consider first the case of no noise, $\varepsilon=0$, and define an absorbing set $A \subseteq \Gamma^{n}$ in the standard way by

(i) for all $\omega \in A$ and for all $\omega^{\prime} \notin A$, the transition probability from $\omega$ to $\omega^{\prime}$ is $p_{\omega \omega^{\prime}}=0$; and

(ii) for all $\omega, \omega^{\prime} \in A$, there exists a finite $m \in \mathbb{N}$ s.t. $p_{\omega \omega^{\prime}}^{(m)}>0, p_{\omega \omega^{\prime}}^{(m)}$ being the $m$-step transition probability from $\omega$ to $\omega^{\prime}$.

Vega-Redondo (1997) showed that a homogeneous population of imitators converges to the competitive solution $\omega^{*}=\left(q_{1}^{*}, \ldots, q_{n}^{*}\right)$ defined by for all $i \in N, q_{i}^{*} p\left(Q^{*}\right)-c\left(q_{i}^{*}\right) \geq q_{i} p\left(Q^{*}\right)-c\left(q_{i}\right)$, for all $q_{i} \in \Gamma$, with $Q^{*}:=\sum_{i \in N} q_{i}^{*}$. Can the competitive solution be an absorbing state given a heterogeneous population of imitators and optimizers? Since $n$ is finite, each optimizers' share on the competitive output is larger than the best response. Hence they will deviate to the best response leading to a state different from the competitive solution. It follows that the competitive solution is not an absorbing state.

Consider now a state where every firm sets its symmetric Cournot Nash equilibrium output.

Definition 4 (Cournot Nash Equilibrium) A state $\omega^{C}=\left(q_{1}^{C}, q_{2}^{C}, \ldots, q_{n}^{C}\right) \in \Gamma^{n}$ is a Cournot Nash equilibrium if for all $i \in N$,

$$
q_{i}^{C} p\left(Q^{C}\right)-c\left(q_{i}^{C}\right) \geq q_{i} p\left(Q^{C}-q_{i}^{C}+q_{i}\right)-c\left(q_{i}\right), \text { for all } q_{i} \in \Gamma
$$

In a heterogeneous population, imitators do not deviate since all firms set identical outputs and earn identical profits. Optimizers do not deviate too since they set their best response quantities anyway. Thus the symmetric Cournot Nash equilibrium is an absorbing state. However, is it the unique absorbing state? Consider the following state:

Definition 5 (Pseudo-Stackelberg Solution) The Pseudo-Stackelberg solution $\omega^{S}=\left(q_{1}, \ldots, q_{\theta n}, q_{\theta n+1}, \ldots, q_{n}\right) \in \Gamma^{n}$ is defined by: ${ }^{4}$

\footnotetext{
${ }^{4}$ Assumption 3 ensures that the best responses are unique (see Lemma 2 in the appendix).
} 
(i) for all $i \in I, q_{i}=q^{S}$ s.t.

$$
q^{S} p\left(\theta n q^{S}+(1-\theta) n q^{O}\right)-c\left(q^{S}\right)>q p\left(\theta n q^{S}+(1-\theta) n q^{O}\right)-c(q), \text { for all } q \neq q^{S}
$$

(ii) for all $i \in N \backslash I, q_{i}=q^{O}$,

$$
q^{O}:=b\left(\theta n q^{S}+((1-\theta) n-1) q^{O}\right)
$$

In the Pseudo-Stackelberg solution all imitators set identical outputs. This output maximizes profits of imitators given that they do not perceive any influence on the price and the optimizers set identical best responses. This outcome has features of the competitive solution (for imitators) and the Cournot Nash equilibrium (for optimizers). If $\theta=1$, then it is identical to the competitive solution since Inequality (9) becomes vacuous. If $\theta=0$, then it is identical to the Cournot Nash equilibrium since Inequality (8) becomes vacuous. We call this outcome the Pseudo-Stackelberg Solution because of its similarity to the Stackelberg solution in the literature. Analogous to the profits of von Stackelberg's (1934) independent and dependent firms, every imitator is strictly better off than is every optimizer since Inequality (8) holds for all $q \in \Gamma, q \neq q^{S}$, hence also for $q^{O} \neq q^{S}$. I.e., it follows that if $q^{O} \neq q^{S}$ then $^{5}$ $\pi_{i}\left(q^{S}, q^{O}, n, \theta\right)>\pi_{j}\left(q^{S}, q^{O}, n, \theta\right)$, for all $i \in I$ and for all $j \in N \backslash I$. Every imitator is strictly better off than every optimizer.

Why is the Pseudo-Stackelberg solution an absorbing state? All imitators set identical outputs and each of them is strictly better off than is any optimizer. Hence an imitator has no reason to deviate from her output. Optimizers do not deviate too from their output since each of them plays the best response. Thus the Pseudo-Stackelberg solution is an absorbing state.

We assume that the Pseudo-Stackelberg solution exists in $\Gamma^{n}$. The existence of PseudoStackelberg solution is analogous to the existence of competitive solution in Vega-Redondo (1997) and the existence of Cournot Nash equilibrium. Standard assumptions on costs, i.e., strictly increasing marginal costs and small fixed costs, and Assumption 3 in the next section suffice.

Previous arguments suggest already that the Cournot Nash equilibrium and the PseudoStackelberg solution may not be the only candidates for solutions. To facilitate the analysis we

\footnotetext{
${ }^{5}$ For notational convenience we write $\pi_{i}\left(q, q^{\prime}, n, \theta\right)$ for $\pi_{i}\left(q, \theta n q+(1-\theta) n q^{\prime}\right)$ if $i \in I$, or for $\pi_{i}\left(q^{\prime}, \theta n q+(1-\theta) n q^{\prime}\right)$ if $i \in N \backslash I$.
} 
define the following set of states:

Definition 6 (Pseudo-Stackelberg States) The set of Pseudo-Stackelberg states $H$ consists of all states $\omega=\left(q_{1}, \ldots, q_{\theta n}, q_{\theta n+1}, \ldots, q_{n}\right) \in \Gamma^{n}$ satisfying the following properties: 6

(i) $q_{i}=q^{I}$, for all $i \in I$ and some $q^{I} \in \Gamma$,

(ii) $q_{i}=q^{O}$, for all $i \in N \backslash I, q^{O}:=b\left(\theta n q^{I}+((1-\theta) n-1) q^{O}\right)$,

(iii) $\pi_{i}\left(q^{I}, q^{O}, n, \theta\right) \geq \pi_{j}\left(q^{I}, q^{O}, n, \theta\right)$, for all $i \in I$ and all $j \in N \backslash I$,

(iv) $\pi_{i}\left(q^{I}, q^{O}, n, \theta\right)=\pi_{j}\left(q^{I}, q^{O}, n, \theta\right)$ for all $i \in I$ and all $j \in N \backslash I$, iff $q^{I}=q^{O}$.

Each Pseudo-Stackelberg state is an absorbing state. If condition (i) is not satisfied, then an imitator may mimic a different output decision from another imitator if the latter happens to have higher profits. If condition (ii) is not satisfied, all optimizers that don't play a best response will have an incentive to deviate. If condition (iii) is not satisfied, imitators will mimic optimizers. To understand the motivation of (iv) note that by symmetry, $q^{I}=q^{O}$ implies $\pi_{i}\left(q^{O}, q^{I}, n, \theta\right)=\pi_{j}\left(q^{O}, q^{I}, n, \theta\right)$ for all $i \in I$ and all $j \in N \backslash I$. To see the purpose of the other direction note that if $\pi_{i}\left(q^{O}, q^{I}, n, \theta\right)=\pi_{j}\left(q^{O}, q^{I}, n, \theta\right)$ for some $i \in I$ and some $j \in N \backslash I$, and $q^{I} \neq q^{O}$ then some imitators would be indifferent between $q^{I}$ and $q^{O}$, thus adding a source of instability.

In each Pseudo-Stackelberg state, imitators are weakly better off than are optimizers. In fact, imitators are strictly better off in any Pseudo-Stackelberg state except the Cournot Nash equilibrium, the only state where optimizers are as well off as imitators.

It is clear that the set of Pseudo-Stackelberg states is nonempty since the Cournot Nash equilibrium - assume that it exists - belongs to it. Moreover, it is easy to see that the competitive solution is not a Pseudo-Stackelberg state since optimizers do not set a best response in the competitive solution (unless $n \rightarrow \infty$ or $\theta=1$ ). Finally, if marginal costs are strictly increasing then the Pseudo-Stackelberg solution is a Pseudo-Stackelberg state.

Can there be other absorbing sets like cycles? Following example shows that there are cases where we can answer this question in the affirmative.

\footnotetext{
${ }^{6}$ Again, Assumption 3 ensures that the best responses are unique (see Lemma 2 in the appendix).
} 
Example 1 (Cycle) Consider two imitators and one optimizer who face a linear demand $p(Q)=10-Q$, and a cost function $c\left(q_{i}\right)=\frac{25}{2}$ for any $q_{i}>0$ and zero otherwise, and let $\Gamma=\{0,5\}$. It is straightforward to verify that the monopoly output is 5 and the monopoly profit is $\frac{25}{2}$. If two firms choose 5 (and the third chooses zero output) then each of the two gets a profit of $-\frac{25}{2}$. Suppose that the two imitators choose 0 while the optimizer chooses 5 . Since the optimizer gets a profit of $\frac{25}{2}$ while the imitators make a 0 profit each, the imitators will have an incentive to mimic the optimizer who will in turn have to adjust his own output. Thus in a new state, each imitator chooses 5 and earns $-\frac{25}{2}$ while the optimizer chooses 0 and makes a 0 profit. Again, the imitators have an incentive to mimic the optimizer. After the optimizer adjusts, we reach again the case where the optimizer chooses 5 and the imitators choose 0.

Example 1 appears to be contrived by the restricted action space. But even with a finer grid there could be cycles. The following two assumptions together with inertia are sufficient to rule out absorbing cycles.

Assumption $2 \theta \leq \frac{1}{2}$.

Assumption 3 For $q_{-i}^{\prime}<q_{-i}, q^{\prime} \in b\left(q_{-i}^{\prime}\right), q \in b\left(q_{-i}\right)$, we have

$$
0>\frac{q^{\prime}-q}{q_{-i}^{\prime}-q_{-i}}>-1
$$

Assumption 3 states that the slopes of the best response correspondence are strictly lower than 0 and strictly larger than -1 . Former implies by Dubey, Haimanko, and Zapechelnyuk (2006) that the game is a (finite) pseudo-potential game and has a Cournot Nash equilibrium. Hence there exists a finite improvement path such that sequential best response converges to the Cournot Nash equilibrium. The Cournot games in the literature are mostly pseudo-potential games.

The assumption that the slopes of the best response correspondence are strictly larger than -1 is made in order to obtain an unique best response. Vives (2000, Theorem 2.8) shows in a simple proof that if a Cournot Nash equilibrium exists and the above assumption holds, then it must be unique. Note that the condition is equivalent to if $q_{-i}^{\prime}<q_{-i}$ then $q_{-i}^{\prime}+q^{\prime}<q_{-i}+q$. It means that total output is strictly increasing in the opponents' output when the player sets best responses. Since we have a symmetric game, the uniqueness condition implies that the unique 
Cournot Nash equilibrium is symmetric (Vives, 2000, Remark 17) and that the best response correspondence is in fact a function (see Vives, 2000, p. 43). Assumption 3 is standard in Cournot games (see Amir, 1996, Amir and Lambson, 2000, and Vives, 2000, section 4.1 and exercise 4.5, for sufficient conditions on demand and costs implying Assumption 3).

\section{Results}

We are finally ready to state our results. Let $Z$ be the collection of all absorbing sets in $\Gamma^{n}$. Recall that $H$ is the set of all Pseudo-Stackelberg states (Definition 6).

Proposition 1 If $\varepsilon=0$ and all previous assumptions hold, then $Z=\{\{\omega\}: \omega \in H\}$.

In previous section, we have argued already that each Pseudo-Stackelberg state must be an absorbing set. To show that every absorbing set is a Pseudo-Stackelberg state, we use Assumptions 2 and 3 to show that there aren't any cycles and any adjustment process converges to a Pseudo-Stackelberg state.

Let $S$ denote the support of the long run distribution $\varphi^{*}$. By standard results, Proposition 1 implies that $S \subseteq H$.

Corollary 1 Under all previous assumptions, in the long run imitators are weakly better off than are optimizers.

The question we answer next is whether the noise selects among absorbing sets.

Proposition 2 If $\theta>0$ and Assumptions 1 and 3 hold, then for any absorbing set $A \neq\left\{\omega^{S}\right\}$ we have $S \neq A$. In particular, $S \neq\left\{\omega^{C}\right\}$.

Since we do not impose Assumption 2 in Proposition 2, we allow for cycles if any. We show in the appendix, Lemma 4, that the Pseudo-Stackelberg solution can be reached from any absorbing set by just one suitable mutation (and the unperturbed adjustment process). This is so because an imitator when switching to the output of the Pseudo-Stackelberg solution may decrease her own payoff but decreases the payoffs of any other players even more. By 
Lemma 3 (see appendix) the instability against a single mutation is sufficient to conclude that any absorbing set $A \neq\left\{\omega^{S}\right\}$ can never be the unique long run outcome. Since the Cournot Nash equilibrium is the only Pseudo-Stackelberg state in which optimizers are as well off as are imitators, it is worth to put following implication on record:

Corollary 2 Under Assumptions 1, 2 and 3, in the long run imitators are strictly better off than are optimizers.

Proposition 2 does not exclude any absorbing sets from the support of the long run distribution. Since in Lemma 4, which is used to prove Proposition 2, the Pseudo-Stackelberg solution figures prominently, one may conjecture that the Pseudo-Stackelberg solution is the unique long run outcome. This would be also analogous to Vega-Redondo's (1997) competitive solution as long run outcome in a homogeneous population of imitators. It turns out that this conjecture is false. An imitator deviating from the Pseudo-Stackelberg solution may become worse off than others and consequently should switch back. However, in the meantime, some optimizers may have already adjusted their best responses. This adjustment may lead temporarily to higher relative profits of those optimizers. Subsequently, imitators may mimic those optimizers such that the adjustment process may lead to another Pseudo-Stackelberg state. The exact support of the long run distribution depends upon the parameters of the game. We show in Example 2 that the support may comprise of the entire set of Pseudo-Stackelberg states (see the appendix for a proof).

Example 2 If $p(Q)=10-Q, c\left(q_{i}\right)=\frac{1000}{501} q_{i}^{2}+1, \theta=\frac{1}{5}, n=5, \delta=0.001$, and $\nu$ is sufficiently large, then $S=H$, i.e., the support of the long run distribution comprises of the entire set of Pseudo-Stackelberg states.

\section{Discussion}

Conlisk (1980) also analyzes a dynamic model with imitators and optimizers. However, he takes the cost of optimizing into account, and this cost is a key for obtaining his results. Our result appears to be stronger since in our work imitators are better off than are optimizers even without any optimizers' cost of sophistication. Conlisk's (1980) result has a similar flavor to 
Stahl (1993), who concludes using a different approach that dumb players may never die out and smart players with maintenance costs may vanish. Droste, Hommes and Tuinstra (2002) consider a large population of firms matched randomly into a Cournot duopoly. A fraction of firms play a best response to the average output in the previous period whereas others perfectly forecast the output of either decision rule, play a best response to the forecast but bear an extra information cost. They show that in an evolutionary dynamics with noise both decision rules survive in the long run with fluctuating fractions. Using a different approach, Banerjee and Weibull (1995) study optimizers and players that are programmed to actions in evolutionary symmetric 2-player games. They show that long run resting states hold a positive share of programmed players. Gale and Rosenthal (1999) study "imitators" and experimenters but their "imitators" differ from ours as they adjust towards the average action adopted by opponents instead imitate the action of the most successful player. Roughly they find that the population converges to the Nash equilibrium in various games with a unique equilibrium. Juang (2002) studies imitators and myopic optimizers in $2 \times 2$ coordination games with a risk-dominant and Pareto-dominant equilibrium. The equilibrium selection depends on the relative frequency of agents using the two rules. Kaarbøe and Tieman (1999) study imitators and myopic optimizers in strict supermodular games and find among others that the set of absorbing sets corresponds to the set of Nash equilibria. This is in contrast with the strict submodular game studied in our paper, for which there are also other absorbing states than the Nash equilibrium. Hehenkamp and Kaarbøe (2007) study one imitator and one myopic optimizer in rapid changing games with strategic substitutes. If the optimizer can adjust decisions as fast as some parameters of the game change (like the demand), then it is possible that the optimizer is better off than the imitator if strategic substitutes are sufficiently weak. Thijssen (2005) studies firms that imitate successful conjectures in Cournot oligopoly. He shows that the competitive outcome is stochastically stable. ${ }^{7}$

Our results highlight the ambiguous semantics of profit maximization in Cournot oligopoly. The optimizers are absolute profit maximizers in regard to their objective but not in terms of the result. Aiming to maximize absolute profit may not be the way to actually achieving the highest relative profit. We show that the standard text book understanding of profit maximizing

\footnotetext{
${ }^{7}$ See Hommes (2006) for an extensive survey of models with heterogeneous decision rules in economics and finance.
} 
firms can not be supported by evolutionary arguments in Cournot oligopoly. A related point was raised by Fershtman and Judd (1987) who showed that if firms are modelled by a principleagent relationship in Cournot oligopoly, then profit maximizing owners may not want managers to maximize profits.

Earlier experimental studies of Cournot oligopoly by Sauermann and Selten (1959) found some support for the convergence to Cournot Nash equilibrium. Recent studies by Huck, Normann, and Oechssler (1999) found support for imitative behavior. Whereas in former subjects had profit tables for an easy calculation of the best response available, in later studies subjects received feedback about the competitors' profits and output levels. The informational framework of these experimental designs corresponds closely to the information required by each of the two decision rules (see also Offerman, Potters, and Sonnemans, 2002). Since both, imitation behavior as well as best response, is supported by experimental findings in Cournot markets, it would be natural to explore our results experimentally by providing different information to different firms. This shall be left to further research. Yet, experimental results by Dürsch et al. (2008) on Cournot duopoly show already that subjects earn on average higher profits when playing against computers programmed to best response but do much worse against computers programmed to imitation.

\section{A Proofs}

\section{Proof of Lemma 1.}

Let $q^{\prime \prime}>q^{\prime}$ and $Q^{\prime \prime}>Q^{\prime}$. Since by Assumption 1, $p$ is strictly decreasing

$$
\begin{aligned}
p\left(Q^{\prime}\right) & >p\left(Q^{\prime \prime}\right) \\
p\left(Q^{\prime}\right)\left(q^{\prime \prime}-q^{\prime}\right) & >p\left(Q^{\prime \prime}\right)\left(q^{\prime \prime}-q^{\prime}\right) \\
p\left(Q^{\prime}\right)\left(q^{\prime \prime}-q^{\prime}\right)-c\left(q^{\prime \prime}\right)+c\left(q^{\prime}\right) & >p\left(Q^{\prime \prime}\right)\left(q^{\prime \prime}-q^{\prime}\right)-c\left(q^{\prime \prime}\right)+c\left(q^{\prime}\right) \\
\pi\left(q^{\prime \prime}, Q^{\prime}\right)-\pi\left(q^{\prime}, Q^{\prime}\right) & >\pi\left(q^{\prime \prime}, Q^{\prime \prime}\right)-\pi\left(q^{\prime}, Q^{\prime \prime}\right)
\end{aligned}
$$

A function satisfying last inequality is called strictly submodular. Strict quasisubmodularity is an immediate implication.

For the proofs of the following results are useful:

Lemma 2 If Assumptions 1 and 3 hold then we conclude the following: 
(i) For any fixed quantities by imitators, the game in which the set of optimizers is the set of players has a Nash equilibrium.

(ii) Sequential best response converges to such Nash equilibrium in finite time.

(iii) Any optimizer's best response is unique, and in above Nash equilibrium best responses are symmetric. ${ }^{8}$

(iv) Given $\theta \in(0,1]$, let $x^{\prime}, x$ be total outputs of all imitators. If $x^{\prime}<x$, then $x^{\prime}+(1-\theta) n q^{O^{\prime}}<$ $x+(1-\theta) n q^{O}$, with $q^{O}=b\left(x+((1-\theta) n-1) q^{O}\right)$ and $q^{O^{\prime}}=b\left(x^{\prime}+((1-\theta) n-1) q^{O^{\prime}}\right)$.

(v) If $\omega^{S}$ exists, then it is unique.

Proof. For any fixed quantities by imitators, the game in which the set of players is the set of optimizers satisfies Assumptions 1 and 3. Hence, (i) and (ii) are implied Dubey, Haimanko, and Zapechelnyuk (2006), and (iii) is implied by Vives (2000, p. 43) for sufficiently small $\delta>0$.

(iv) For $\theta=1$, the result follows trivially. Consider now $\theta \in(0,1)$. Suppose to the contrary that $x^{\prime}<x$ and $x^{\prime}+(1-\theta) n q^{O^{\prime}} \geq x+(1-\theta) n q^{O}$. Last inequality is equivalent to $x^{\prime}-x \geq(1-\theta) n\left(q^{O}-q^{O^{\prime}}\right)$. Since by assumption $0>x^{\prime}-x$ we conclude that $0>(1-\theta) n\left(q^{O}-q^{O^{\prime}}\right)$. Since $\theta \in(0,1)$ and $n \geq 1$, the last equality is satisfied if and only if $0>q^{O}-q^{O^{\prime}}$. Define $q_{-i}^{\prime}:=x^{\prime}+((1-\theta) n-1) q^{O^{\prime}}$ and analogously for $q_{-i}$. Suppose $q_{-i}^{\prime} \geq q_{-i}$, then by Assumption 3 (strictly decreasing best responses) $q^{O^{\prime}} \leq q^{O}$ (with equality if $q_{-i}^{\prime}=q_{-i}$ ), a contradiction to above. Suppose now that we have $q_{-i}^{\prime}<q_{-i}$. Then by Assumption 3, $q_{-i}^{\prime}+q^{O^{\prime}}<q_{-i}+q^{O}$, a contradiction to $x^{\prime}+(1-\theta) n q^{O^{\prime}} \geq x+(1-\theta) n q^{O}$ above.

(v) Let $\omega^{S^{\prime}}$ and $\omega^{S^{\prime \prime}}$ be two Pseudo-Stackelberg solutions with $\omega^{S^{\prime}} \neq \omega^{S^{\prime \prime}}$. Denote by $Q^{S^{\prime}}=$ $\theta n q^{S^{\prime}}+(1-\theta) n q^{O^{\prime}}, Q^{S^{\prime \prime}}=\theta n q^{S^{\prime \prime}}+(1-\theta) n q^{O^{\prime \prime}}, q^{O^{\prime}}=b\left(\theta n q^{S^{\prime}}+((1-\theta) n-1) q^{O^{\prime}}\right)$, and $q^{O^{\prime \prime}}=$ $b\left(\theta n q^{S^{\prime \prime}}+((1-\theta) n-1) q^{O^{\prime \prime}}\right)$. Inequality (8) implies $\pi_{i}\left(q^{S^{\prime}}, Q^{S^{\prime}}\right)>\pi_{i}\left(q^{S^{\prime \prime}}, Q^{S^{\prime}}\right)$ and $\pi_{i}\left(q^{S^{\prime \prime}}, Q^{S^{\prime \prime}}\right)>$ $\pi_{i}\left(q^{S^{\prime}}, Q^{S^{\prime \prime}}\right)$. If $q^{S^{\prime \prime}}>q^{S^{\prime}}$ then $Q^{S^{\prime \prime}}>Q^{S^{\prime}}$ by (iv). By Assumption 1 (Lemma 1, upper Formula (2)) $\pi_{i}\left(q^{S^{\prime \prime}}, Q^{S^{\prime \prime}}\right)>\pi_{i}\left(q^{S^{\prime}}, Q^{S^{\prime \prime}}\right)$ implies $\pi_{i}\left(q^{S^{\prime \prime}}, Q^{S^{\prime}}\right)>\pi_{i}\left(q^{S^{\prime}}, Q^{S^{\prime}}\right)$, a contradiction. Likewise for $q^{S^{\prime \prime}}<q^{S^{\prime}}$ (using lower Formula (3)).

\section{Proof of Proposition 1.}

Recall that $Z$ is the collection of all absorbing sets for $\varepsilon=0$. We need to show that Assumptions 1 to 2 imply $Z=\{\{\omega\}: \omega \in H\}$.

First, we show that every $\{\omega\}$ with $\omega \in H$ is an absorbing state. Since in $\omega \in H$ it follows by Definition 6 (i), (iii), and (iv) that no imitator $i \in I$ wants to deviate form its output in $\omega \in H$. Since $\omega \in H$, it follows by aforesaid Definition 6 (ii) that no optimizer $i \in N \backslash I$ wants to deviate from its best

\footnotetext{
${ }^{8}$ This implies that the Cournot Nash equilibrium is unique and symmetric.
} 
response in $\omega \in H$, which by Lemma 2 is uniquely defined. Since both types of firms do not deviate in $\omega \in H$, no firm $i \in N$ deviates in any of the following periods. Hence $\{\omega\}$ is an absorbing state.

Second, we show that there are no other absorbing sets other than $\{\{\omega\}: \omega \in H\}$. Consider any state $\omega^{\prime} \notin H$. At least one condition of (i) to (iv) of Definition 6 is violated. From any $\omega^{\prime} \notin H$ there is an unperturbed adjustment path based on the decision rules leading in the subsequent periods to a state $\tilde{\omega}=\left(q^{I}, \stackrel{\theta n}{\cdots}, q^{I}, q^{O},{ }^{(1-\theta) n}, q^{O}\right)$ in which (a) all imitators play identical quantities $q^{I}$ and (b) all optimizers play identical best responses $q^{O}=b\left(\theta n q^{I}+((1-\theta) n-1) q^{O}\right)$. (a) follows from the imitators' decision rule (Definition 2) since by inertia there is positive probably that in the next period all imitators adjust to the same quantity. (b) follows by Assumption 3 (Lemma 2), since each optimizer can reach in finite time the symmetric best response given that imitators play $\theta n q^{I}$. Note that $\tilde{\omega}$ satisfies condition (i) and (ii) of Definition 6. If (iii) or (iv) are satisfied too, then $\tilde{\omega} \in H$. Otherwise, if $\pi_{i}\left(q^{I}, \theta n q^{I}+(1-\theta) n q^{O}\right) \leq \pi_{i}\left(q^{O}, \theta n q^{I}+(1-\theta) n q^{O}\right)$ and $q^{I} \neq q^{O}$, then by previous arguments there exists an unperturbed adjustment path based on the decision rules leading in the subsequent periods to a state $\tilde{\omega}^{\prime}=\left(q^{O}, \ldots n, q^{O}, q^{O^{\prime}},{ }^{(1-\theta) n}, q^{O^{\prime}}\right)$ with $q^{O^{\prime}}=b\left(\theta n q^{O}+((1-\theta) n-1) q^{O^{\prime}}\right)$. Again, $\tilde{\omega}^{\prime}$ satisfies (i) and (ii) but may not satisfy (iii) and (iv) of Definition 6. More generally, for $t=0,1, \ldots$, define inductively states $\tilde{\omega}(t)=\left(q^{t}, \stackrel{\theta n}{\cdots}, q^{t}, q^{t+1}, \stackrel{(1-\theta) n}{\cdots}, q^{t+1}\right)$ by $q^{0}=q^{I}$ and $q^{t+1}=b\left(\theta n q^{t}+((1-\theta) n-1) q^{t+1}\right)$. We need to show that there exists a finite $\bar{t}$ such that $\tilde{\omega}(\bar{t}) \in H$.

Claim: $q^{t} \leq q^{C} \leq q^{t+1}$ or $q^{t+1} \leq q^{C} \leq q^{t}$. Assume $q^{t}, q^{t+1}<q^{C}$. Since $\theta n q^{t}+((1-\theta) n-1) q^{t+1}<$ $(n-1) q^{C}$ it follows from Assumption 3 that $q^{t+1}>q^{C}$, a contradiction. The case $q^{t}, q^{t+1}>q^{C}$ yields a contradiction by analogous arguments.

W.l.o.g. assume $q^{t+2}<q^{C}<q^{t+1}$. We distinguish the cases in Table 1: Case 1 yields a contradiction since $q^{t+2}>q^{t+4}$ and $q^{t+3}>q^{t+5}$ must imply $\theta n q^{t+2}+((1-\theta) n-1) q^{t+3}>\theta n q^{t+4}+((1-\theta) n-1) q^{t+5}$. Exactly the same argument applies to case 4. An analogous argument applies to cases 2 and 3 . We are just left with cases 5 and 6 . Together with above Claim, it implies that along a sequence of states $\tilde{\omega}(t), \tilde{\omega}(t+1), \ldots$, the quantities' distances to $q^{C}$ either only (weakly) increase or only (weakly) decrease. In either case, since $\Gamma$ is finite we must eventually reach a 2-cycle (cycles with higher periodicity are ruled out by the monotonicity of cases 5 and 6 ) or a state in $H$. In a 2-cycle, $\tilde{\omega}(t)=\tilde{\omega}(t+2 k)$ for some $t$ and $k=1,2, \ldots$. In such a cycle we must have

$$
\begin{aligned}
\pi_{i}\left(q^{t+1}, \theta n q^{t}+(1-\theta) n q^{t+1}\right) & \geq \pi_{i}\left(q^{t}, \theta n q^{t}+(1-\theta) n q^{t+1}\right) \\
\pi_{i}\left(q^{t}, \theta n q^{t+1}+(1-\theta) n q^{t}\right) & \geq \pi_{i}\left(q^{t+1}, \theta n q^{t+1}+(1-\theta) n q^{t}\right)
\end{aligned}
$$

W.l.o.g. assume $q^{t+1}>q^{t}$. From Assumption 3 follows $\theta n q^{t}+((1-\theta) n-1) q^{t+1}<\theta n q^{t+1}+((1-\theta) n-1) q^{t}$. Applying Assumption 3 again yields

$$
q^{t}-q^{t+1}>\theta n q^{t}+((1-\theta) n-1) q^{t+1}-\theta n q^{t+1}-((1-\theta) n-1) q^{t}
$$


Table 1: Cases

\begin{tabular}{|c|c|c|}
\hline Case & If & then Assumption 3 implies \\
\hline 1 & $\begin{array}{l}q^{t+1}>q^{t+3}>q^{t+5} \\
q^{t+2}>q^{t+4}>q^{t+6}\end{array}$ & $\begin{array}{c}\theta n q^{t}+((1-\theta) n-1) q^{t+1}<\theta n q^{t+2}+((1-\theta) n-1) q^{t+3}<\theta n q^{t+4}+((1-\theta) n-1) q^{t+5} \\
\theta n q^{t+1}+((1-\theta) n-1) q^{t+2}<\theta n q^{t+3}+((1-\theta) n-1) q^{t+4}<\theta n q^{t+5}+((1-\theta) n-1) q^{t+6}\end{array}$ \\
\hline 2 & $\begin{array}{l}q^{t+1}<q^{t+3}<q^{t+5} \\
q^{t+2}<q^{t+4}<q^{t+6}\end{array}$ & $\begin{array}{c}\theta n q^{t}+((1-\theta) n-1) q^{t+1}>\theta n q^{t+2}+((1-\theta) n-1) q^{t+3}>\theta n q^{t+4}+((1-\theta) n-1) q^{t+5} \\
\theta n q^{t+1}+((1-\theta) n-1) q^{t+2}>\theta n q^{t+3}+((1-\theta) n-1) q^{t+4}>\theta n q^{t+5}+((1-\theta) n-1) q^{t+6}\end{array}$ \\
\hline 3 & $\begin{array}{l}q^{t+1}>q^{t+3}<q^{t+5} \\
q^{t+2}<q^{t+4}>q^{t+6}\end{array}$ & $\begin{array}{c}\theta n q^{t}+((1-\theta) n-1) q^{t+1}<\theta n q^{t+2}+((1-\theta) n-1) q^{t+3}>\theta n q^{t+4}+((1-\theta) n-1) q^{t+5} \\
\theta n q^{t+1}+((1-\theta) n-1) q^{t+2}>\theta n q^{t+3}+((1-\theta) n-1) q^{t+4}<\theta n q^{t+5}+((1-\theta) n-1) q^{t+6}\end{array}$ \\
\hline 4 & $\begin{array}{l}q^{t+1}<q^{t+3}>q^{t+5} \\
q^{t+2}>q^{t+4}<q^{t+6}\end{array}$ & $\begin{array}{c}\theta n q^{t}+((1-\theta) n-1) q^{t+1}>\theta n q^{t+2}+((1-\theta) n-1) q^{t+3}<\theta n q^{t+4}+((1-\theta) n-1) q^{t+5} \\
\theta n q^{t+1}+((1-\theta) n-1) q^{t+2}<\theta n q^{t+3}+((1-\theta) n-1) q^{t+4}>\theta n q^{t+5}+((1-\theta) n-1) q^{t+6}\end{array}$ \\
\hline 5 & $\begin{array}{l}q^{t+1}>q^{t+3}>q^{t+5} \\
q^{t+2}<q^{t+4}<q^{t+6}\end{array}$ & $\begin{array}{c}\theta n q^{t}+((1-\theta) n-1) q^{t+1}<\theta n q^{t+2}+((1-\theta) n-1) q^{t+3}<\theta n q^{t+4}+((1-\theta) n-1) q^{t+5} \\
\theta n q^{t+1}+((1-\theta) n-1) q^{t+2}>\theta n q^{t+3}+((1-\theta) n-1) q^{t+4}>\theta n q^{t+5}+((1-\theta) n-1) q^{t+6}\end{array}$ \\
\hline 6 & $\begin{array}{l}q^{t+1}<q^{t+3}<q^{t+5} \\
q^{t+2}>q^{t+4}>q^{t+6}\end{array}$ & $\begin{array}{c}\theta n q^{t}+((1-\theta) n-1) q^{t+1}>\theta n q^{t+2}+((1-\theta) n-1) q^{t+3}>\theta n q^{t+4}+((1-\theta) n-1) q^{t+5} \\
\theta n q^{t+1}+((1-\theta) n-1) q^{t+2}<\theta n q^{t+3}+((1-\theta) n-1) q^{t+4}<\theta n q^{t+5}+((1-\theta) n-1) q^{t+6}\end{array}$ \\
\hline & & $>\quad \frac{1}{2}$ \\
\hline
\end{tabular}

a contradiction to Assumption 2. This completes the proof of Proposition 1.

In order to characterize the support of the unique limiting invariant distribution, we consider small perturbations, $\varepsilon>0$. We call states $\omega$ and $\omega^{\prime}$ adjacent if exactly one mutation can change the state from $\omega$ to $\omega^{\prime}$ (and vice versa), i.e., if exactly one firm's change of output changes the state $\omega$ to the state $\omega^{\prime}$. The set of all states adjacent to the state $\omega$ is the single mutation neighborhood of $\omega$ denoted by $M(\omega)$. The basin of attraction of an absorbing set $A$ is the set $B(A)=\left\{\omega \in \Gamma^{n} \mid \exists m \in \mathbb{N}, \exists \omega^{\prime} \in A\right.$ s.t. $\left.p_{\omega \omega^{\prime}}^{(m)}>0\right\}$. A recurrent set $R$ is a minimal collection of absorbing sets with the property that there do not exist absorbing sets $A \in R$ and $A^{\prime} \notin R$ such that there exists an $\omega \in A, M(\omega) \cap B\left(A^{\prime}\right) \neq \emptyset$.

Lemma 3 Given a regularly perturbed finite Markov chain, then at least one recurrent set exists. Recurrent sets are disjoint. Let the state $\omega$ be contained in the support of the unique limiting invariant distribution $\varphi^{*}$. Then $\omega \in R, R$ being a recurrent set. Moreover, for all $\omega^{\prime} \in R, \varphi^{*}\left(\omega^{\prime}\right)>0$.

See Samuelson (1997), Lemma 7.1 and Proposition 7.7., proof pp. 236-238, for a proof.

\section{Proof of Proposition 2.}


Lemma 4 Suppose Assumptions 1 and 3 hold. If $\omega^{S} \in H$ then for any absorbing set $A \neq\left\{\omega^{S}\right\}$ there exist $\omega \in A$ such that $M(\omega) \cap B\left(\left\{\omega^{S}\right\}\right) \neq \emptyset$.

Proof of Lemma. Assume $\omega^{S} \in H$. In the proof of Proposition 1 we showed that any absorbing set (no matter whether it is a singleton or not) contains a state such that for some $q \in \Gamma, \tilde{\omega}=(q, . . n$ $\left., q, q^{O},(1-\theta) n, q^{O}\right)$ with $q^{O}=b\left(\theta n q+((1-\theta) n-1) q^{O}\right)$. It is sufficient to show that for any $q \in \Gamma \backslash\left\{q^{S}\right\}$, $k \in \mathbb{N}, 0<k \leq \theta n$,

$$
q^{S} p\left((\theta n-k) q+k q^{S}+(1-\theta) n q^{O}\right)-c\left(q^{S}\right)>q p\left((\theta n-k) q+k q^{S}+(1-\theta) n q^{O}\right)-c(q),
$$

with $q^{O}=b\left((\theta n-k) q+k q^{S}+((1-\theta) n-1) q^{O}\right)$.

By Assumption 1, Lemma 1, $\pi_{i}$ is strictly quasisubmodular (Formulas $(2)$ and $(3)$ ) in $(q, Q)$ on $\Gamma \times\{0, \delta, 2 \delta, \ldots, n \nu \delta\}$. Set $q^{\prime \prime} \equiv q^{S}, q^{\prime} \equiv q, Q^{\prime}=(\theta n-k) q+k q^{S}+(1-\theta) n q^{O^{\prime}}$ and $Q^{\prime \prime}=\theta n q^{S}+(1-\theta) n q^{O^{\prime \prime}}$ with $q^{O^{\prime}} \equiv q^{O}$ and $q^{O^{\prime \prime}} \equiv b\left(\theta n q^{S}+((1-\theta) n-1) q^{O^{\prime \prime}}\right)$ being uniquely defined by Lemma 2. If $q^{\prime \prime}>q^{\prime}$ then $\theta n q^{\prime \prime}>(\theta n-k) q^{\prime}+k q^{\prime \prime}$. By Lemma 2 (iv), we conclude that $Q^{\prime \prime}>Q^{\prime}$. If $q^{\prime \prime}<q^{\prime}$ then $\theta n q^{\prime \prime}<(\theta n-k) q^{\prime}+k q^{\prime \prime}$. By Lemma 2 (iv), we conclude that $Q^{\prime \prime}<Q^{\prime}$. It follows that if $q^{S}>q$ then the

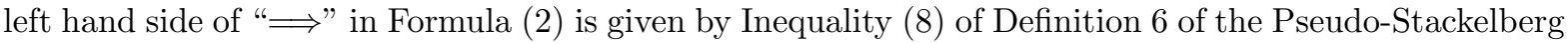
solution (i). In this case the right hand side of " $\Longrightarrow$ " in Formula (2) yields above Inequality (15). If

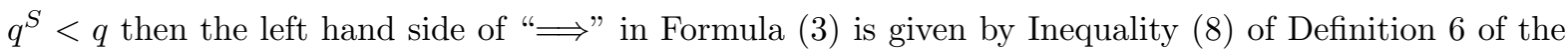
Pseudo-Stackelberg solution (i). In this case the right hand side of " $\Longrightarrow$ " in Formula (3) yields above Inequality (15). Finally, set $k=1$ to see that one suitable mutation only is required to connect every absorbing set to $\omega^{S} \in H$.

Since Lemma 4 holds for any absorbing set except the Pseudo-Stackelberg solution, it holds also for the Cournot Nash equilibrium $\omega^{C}$. From Lemma 3 follows that Lemma 4 implies Proposition 2.

\section{Proof of Example 2.}

We show that in Example 2 we connect all Pseudo-Stackelberg states by a sequence of single suitable mutations. Denote by $\bar{\omega}$ (resp. $\underline{\omega}$ ) the Pseudo-Stackelberg state with the largest (resp. smallest) symmetric output of the imitators. I.e., if $\bar{q}^{I}$ is the symmetric output of any imitator in $\bar{\omega}$, there should not exist $\omega \in H$ s.t. $q_{\omega}^{I}>\bar{q}^{I}$ (analogously for $\underline{\omega}$ ). Let $q_{\omega_{j}}^{I}$ be the identical output of imitators in the state $\omega_{j} \in H$. We call a sequence of Pseudo-Stackelberg states $\omega_{1}, \ldots, \omega_{m} \in H$ increasing (resp. decreasing) if and only the identical output of each imitator in those Pseudo-Stackelberg states is ordered such that $q_{\omega_{j}}^{I}<q_{\omega_{j+1}}^{I}\left(\right.$ resp. $\left.q_{\omega_{j}}^{I}>q_{\omega_{j+1}}^{I}\right), j=1, \ldots, m-1$. Such order on $H$ is the natural order on $\Gamma$.

We say that $\pi_{i}$ is quasiconcave in $q_{i}$ on $\Gamma$ for all $Q \in\{0, \delta, 2 \delta, \ldots, n \nu \delta\}$, i.e., for any $q_{i}, q_{i}^{\prime}, q_{i}^{\prime \prime} \in \Gamma$ with 
$q_{i}^{\prime} \leq q_{i} \leq q_{i}^{\prime \prime}$,

$$
\pi_{i}\left(q_{i}, Q\right) \geq \min \left\{\pi_{i}\left(q_{i}^{\prime}, Q\right), \pi\left(q_{i}^{\prime \prime}, Q\right)\right\}, \text { for all } Q \in\{0, \delta, 2 \delta, \ldots, n \nu \delta\}
$$

Lemma 5 Suppose that Assumptions 1 and 3 hold and that $\pi_{i}$ is quasiconcave.

(i) If $\omega^{S} \in H$, then there exists an increasing sequence $\omega_{1}, \ldots, \omega_{m} \in H$ with $\omega_{1}=\underline{\omega}$ and $\omega_{m}=\omega^{S}$ s.t. $M\left(\omega_{j}\right) \cap B\left(\left\{\omega_{j+1}\right\}\right) \neq \emptyset, j=1, \ldots, m-1$.

(ii) If $\omega^{S} \in H$, then there exists a decreasing sequence $\omega_{1}, \ldots, \omega_{m} \in H$ with $\omega_{1}=\bar{\omega}$ and $\omega_{m}=\omega^{S}$ s.t. $M\left(\omega_{j}\right) \cap B\left(\left\{\omega_{j+1}\right\}\right) \neq \emptyset, j=1, \ldots, m-1$.

Proof of Lemma. (i): Let $\omega_{1}, \ldots, \omega_{m} \in H$ be an increasing sequence of absorbing states with $\omega_{1}=\omega^{C}$ and $\omega_{m}=\omega^{S}$. In order to show that $M\left(\omega_{j}\right) \cap B\left(\left\{\omega_{j+1}\right\}\right) \neq \emptyset$ for $j=1, \ldots, m-1$, it is sufficient to show for $0<k<\theta n$

$$
\begin{array}{r}
q_{\omega_{j+1}}^{I} p\left((\theta n-k) q_{\omega_{j}}^{I}+k q_{\omega_{j+1}}^{I}+(1-\theta) n q^{O}\right)-c\left(q_{\omega_{j+1}}^{I}\right)> \\
q_{\omega_{j}}^{I} p\left((\theta n-k) q_{\omega_{j}}^{I}+k q_{\omega_{j+1}}^{I}+(1-\theta) n q^{O}\right)-c\left(q_{\omega_{j}}^{I}\right),
\end{array}
$$

with $q^{O}=b\left((\theta n-k) q_{\omega_{j}}^{I}+k q_{\omega_{j+1}}^{I}+((1-\theta) n-1) q^{O}\right)$, which is uniquely defined by Lemma 2 .

By Assumption 1, Lemma 1, $\pi_{i}$ is strictly quasisubmodular. Set $q^{\prime \prime}=q_{\omega_{j+1}}^{I}, q^{\prime}=q_{\omega_{j}}^{I}, Q^{\prime}=$ $(\theta n-k) q_{\omega_{j}}^{I}+k q_{\omega_{j+1}}^{I}+(1-\theta) n q^{O^{\prime}}$ and $Q^{\prime \prime}=\theta n q_{\omega_{j+1}}^{I}+(1-\theta) n q^{O^{\prime \prime}}$, with $q^{O^{\prime}}=q^{O}$ and $q^{O^{\prime \prime}}=$ $b\left(\theta n q_{\omega_{j+1}}^{I}+((1-\theta) n-1) q^{O^{\prime \prime}}\right)$ being uniquely defined by Lemma 2 .

Note that each $q_{\omega_{j+1}}^{I} \in\left[\underline{q}^{I}, q^{S}\right] \cap \Gamma$. We claim that $\pi_{i}\left(q_{\omega_{j}}^{I}, Q^{\prime \prime}\right)=\min \left\{\pi_{i}\left(q^{S}, Q^{\prime \prime}\right), \pi_{i}\left(q_{\omega_{j}}^{I}, Q^{\prime \prime}\right)\right\}$. We know that $\pi_{i}\left(q^{S}, Q^{S}\right)>\pi_{i}\left(q_{\omega_{j}}^{I}, Q^{S}\right)$ by definition. Since $q_{\omega_{j}}^{I}<q^{S}$ for $j=1, \ldots, m-1$, we know by Lemma 2 that $Q^{S}>Q^{\prime \prime}$. By strict quasisubmodularity (upper Formula $\left.(2)\right) \pi_{i}\left(q^{S}, Q^{\prime \prime}\right)>\pi_{i}\left(q_{\omega_{j}}^{I}, Q^{\prime \prime}\right)$ and the claim follows. By quasiconcavity $\pi_{i}\left(q_{\omega_{j+1}}^{I}, Q^{\prime \prime}\right) \geq \pi_{i}\left(q_{\omega_{j}}^{I}, Q^{\prime \prime}\right)$. It implies Inequality (17) by strict quasisubmodularity (upper Formula (2)). (i) follows from setting $k=1$ in Inequality (17).

(ii) The proof is analogous to (i). Set $q^{\prime}=q_{\omega_{j+1}}^{I}, q^{\prime \prime}=q_{\omega_{j}}^{I}, Q^{\prime \prime}=(\theta n-k) q_{\omega_{j}}^{I}+k q_{\omega_{j+1}}^{I}+(1-\theta) n q^{O^{\prime \prime}}$ and $Q^{\prime}=\theta n q_{\omega_{j+1}}^{I}+(1-\theta) n q^{O^{\prime}}$, with $q^{O^{\prime \prime}}=q^{O}$ and $q^{O^{\prime}}=b\left(\theta n q_{\omega_{j+1}}^{I}+((1-\theta) n-1) q^{O^{\prime}}\right)$ and use the lower Formula (3) of strict quasisubmodularity.

Lemma 6 Let $q^{C 2} \in \Gamma$ be a firm's Cournot duopoly equilibrium output. ${ }^{9}$ There exist $p, c, \theta, \delta$, $\nu$, and finite $n$ such that

$$
q^{C} p\left((2 n-3) q^{C}\right)-c\left(q^{C}\right) \leq 0
$$

\footnotetext{
${ }^{9}$ The Cournot duopoly equilibrium is a special case of the Cournot Nash equilibrium (Definition 4 ) for $n=2$.
} 


$$
\begin{array}{r}
(n-1) q^{C} p\left((n-1) q^{C}\right)-c\left((n-1) q^{C}\right) \leq 0, \\
\pi(q, \nu \delta)<0, \forall q>0, \\
q^{C 2}=\bar{q}^{I}, \\
\underline{q}^{I}=q^{C},
\end{array}
$$

$\pi_{i}$ is quasiconcave in $q_{i}, \omega^{S} \in \Gamma^{n}$ exists, and all previous assumptions are satisfied.

Proof of Lemma. Consider $p, c, \theta, \delta, \nu$, and $n$ in Example 2, i.e., $p(Q)=10-Q, c\left(q_{i}\right)=\frac{1000}{501} q_{i}^{2}+1$, $\theta=0.2, \delta=0.001, n=5$, and sufficiently large $\nu$ (e.g. $\nu=30000$ ). Straight forward calculations verify that Formulas (18) to (22) as well as all previous assumptions hold, and that $\omega^{S}$ exists. Moreover, since $\pi_{i}$ is strictly concave in $q_{i}$, it is quasiconcave.

Lemma 7 Let $p, c, \theta, \delta, \nu$, and $n$ be such that the properties of Lemma 6 hold. Then

(i) $M(\omega) \cap B(\{\underline{\omega}\}) \neq \emptyset$, for all $\omega \in H$,

(ii) $M(\omega) \cap B(\{\bar{\omega}\}) \neq \emptyset$, for all $\omega \in H$.

Proof of Lemma. (i) Suppose in $t$ any arbitrary state $\omega(t) \in H$. By Lemma 2 such state exists. W.l.o.g. suppose that in $t+1$ a mutation by one firm $i \in N$ occurs such that $q_{i}(t+1)=(n-1) q^{C}$. Note that by Lemma 2, the Cournot Nash equilibrium output $q^{C} \in \Gamma$ exists and is unique. Since $\omega(t) \in H$, we have $Q(t+1) \geq(n-1) q^{C}+(n-1) q^{C}=(2 n-2) q^{C}>(2 n-3) q^{C}$. By Lemma 6, Inequality $(18), \pi_{j}(t+1)<0$, for all $j \in N$. W.l.o.g. assume that a firm $k \in N \backslash I, k \neq i$ and only a firm $k$ has the opportunity to adjust output in $t+2$. Since $D_{O}(t+1)=0$, we have $q_{k}(t+2)=0 . Q(t+2) \geq(2 n-3) q^{C}$. By Lemma 6 , Inequality (18), $\pi_{j}(t+1)<0$, for all $j \in N \backslash\{k\}$. Since $D_{O}(t+2)=D_{I}(t+2)=0$ assume w.l.o.g. that all $j \in N \backslash\{i\}$ adjust output in $t+3$ such that $Q(t+3)=q_{i}(t+3)=q_{i}(t+2)=q_{i}(t+1)=(n-1) q^{C}$. By Lemma 6, Inequality $(19), \pi_{i}(t+3) \leq 0$. Since $D_{O}(t+3)=b\left((n-1) q^{C}\right)=q^{C}$ assume w.l.o.g. that another firm $k \in N \backslash I$ has the opportunity to adjust output in $t+4$. Since $\pi_{k}(t+4)>\pi_{j}(t+4)$, $j \in N \backslash\{k\}$ and $D_{I}(t+4)=q^{C}$ we can assume w.l.o.g. that all $j \in I$ adjust output. Let all remaining optimizers adjust output in the subsequent periods such that with positive probability $\omega^{C}$ is reached in finite time (by Lemma 2). By Lemma $6, \omega^{C}=\underline{\omega}$. Since we started in any arbitrary absorbing state $\omega(t) \in H$ (in particular it also includes $\omega^{S}$ if $\omega^{S} \in H$ ) we have shown that $M(A) \cap B(\{\underline{\omega}\}) \neq \emptyset$, for all $A \in Z$.

(ii) Suppose in $t$ any arbitrary state $\omega(t) \in H$. W.l.o.g. assume that in $t+1$ a mutation by an imitator $i \in I$ occurs setting a large quantity $\nu \delta$ such that by Lemma 6 , Inequality $(20), \pi_{j}(t+1)<0$, for all $j \in N$. W.l.o.g. assume that all optimizers in $N \backslash I$ have the opportunity to adjust output in $t+2$. Since $D_{O}(t+1)=0$, we have $q^{O}(t+2)=0$ with $\pi^{O}(t+2)=0$. By Inequality (20), 
we have $\pi^{I}(t+2)<\pi^{O}(t+2)$. W.l.o.g. assume that all imitators in $I$ adjust output in $t+3$ to $D_{I}(t+2)=q^{I}(t+3)=0$. Hence, $Q(t+3)=0$. W.l.o.g. assume now that in $t+4$ two optimizers and only two optimizers adjust output such that by Lemma 2 we reach a market output of $2 q^{C 2}$ in finite time, i.e. at $t+k$. W.l.o.g. assume that in the following period all imitators in $I$ adjust output such that $D_{I}(t+k)=q^{C 2}(t+k+1)$. Let all optimizers in $N \backslash I$ adjust output in subsequent periods such that by Lemma 2 a state $\omega^{C 2}=\left(q_{1}^{C 2}, \ldots, q_{\theta n}^{C 2}, q_{\theta n+1}^{O}, \ldots, q_{n}^{O}\right)$ is reached in finite time. Since by Lemma 6 , $\bar{q}^{I}=q^{C 2}$, we have that $\omega^{C 2}=\bar{\omega}$.

From Lemmata 5 to 7 follows that in Example 2 there exists a sequence of single suitable mutations by which we can move through the entire set of Pseudo-Stackelberg states. It follows that $H$ is the unique recurrent set. By Lemma 3 it follows that $S=H$. This completes the proof of Example 2.

\section{References}

[1] Alchian, A. A., 1950. Uncertainty, evolution, and economic theory, Journal of Political Economy $58,211-221$.

[2] Alós-Ferrer, C., 2004. Cournot versus Walras in dynamic oligopolies with memory, International Journal of Industrial Organization 22, 193-217.

[3] Amir, R., 1996. Cournot oligopoly and the theory of supermodular games, Games and Economic Behavior 15, 132-148.

[4] Amir, R. and Lambson, V.E., 2000. On the effect of entry in Cournot markets, Review of Economic Studies 67, 235-254.

[5] Banerjee, A. and Weibull, J., 1995. Evolutionary selection and rational behavior, in Kirmann, A. and Salmon, M. (Eds.), Learning and rationality in economics, Basil Blackwell, Oxford.

[6] Conlisk, J., 1980. Costly optimizers versus cheap imitators, Journal of Economic Behavior and Organization 1, 275-293.

[7] Droste, E., Hommes, C. and Tuinstra, J., 2002. Endogenous fluctations under evolutionary pressure in Cournot competition, Games and Economic Behavior, 40, 232-269.

[8] Dubey, P., Haimanko, O. and Zapechelnyuk, A., 2006. Strategic complements and substitutes, and potential games, Games and Economic Behavior 54, 77-94.

[9] Dürsch, P., Kolb, A., Oechssler, J. and Schipper, B. C., 2005. Rage against the machines: How subjects learn to play against computers, University of California, Davis. 
[10] Fershtman, C. and Judd, K., 1987. Equilibrium incentives in oligopoly, American Economic Review, $77,927-940$.

[11] Friedman, M., 1953. Essays in positive economics, University of Chicago Press, Chicago.

[12] Gale, D. and Rosenthal, R. W., 1999. Experimentation, imitation, and stochastic stability, Jourmal of Economic Theory 84, 1-40.

[13] Hehenkamp, B. and Kaarbøe, O., 2007. Imitators and optimizers in a changing environment, Journal of Economic Dynamics and Control, forthcoming.

[14] Hommes, C. (2006). Heterogeneous agent models in economics and finance, in: Tesfatsion, L. and Judd, K. (Eds.), Handbook of computational economics, Vol. 2, Agent-based computational economics, Elsevier, Amsterdam, 1109-1186.

[15] Huck, S., Normann, H.-T. and Oechssler, J., 1999. Learning in Cournot oligopoly - An experiment, Economic Journal 109, C80-C95.

[16] Juang W. T., 2002. Rule evolution and equilibrium selection, Games and Economic Behavior 39, $71-90$.

[17] Kaarbøe, O. and Tieman, A., 1999. Equilibrium selection under different learning modes in supermodular games, mimeo., University of Bergen.

[18] Koopmans, T., 1957. Three essays on the state of economic science, McGraw-Hill Book Company.

[19] Masaaki, K., 1997. Markov processes for stochastic modeling, Chapman \& Hall, London.

[20] Milgrom, P. and Shannon, C., 1994. Monotone comparative statics, Econometrica 62, 157-180.

[21] Offerman, T., Potters, J. and Sonnemans, J., 2002. Imitation and belief learning in an oligopoly experiment, Review of Economic Studies 69, 973-997.

[22] Rhode, P. and Stegeman, M., 2001. Non-Nash equilibria of darwinian dynamics with applications to duopoly, International Journal of Industrial Organization 19, 415-453.

[23] Samuelson, L., 1997. Evolutionary games and equilibrium selection, The MIT Press, Cambridge, M.A.

[24] Sauermann, H. and Selten, R., 1959. Ein Oligopolexperiment, Zeitschrift für die gesamten Staatswissenschaften 115, 427-471.

[25] Schaffer, M. E., 1989. Are profit-maximizers the best survivors?, Journal of Economic Behavior and Organization 12, 29-45.

[26] Schipper, B. C., 2003. Submodularity and the evolution of Walrasian behavior, International Journal of Game Theory 32, 471-477. 
[27] von Stackelberg, H., 1934. Marktform und Gleichgewicht, Verlag von Julius Springer, Wien.

[28] Stahl, D., 1993. Evolution of smartn players, Games and Economic Behavior, 5, 604-617.

[29] Thijssen, J., 2005. Nearly-complete decomposability and stochastic stability with an application to Cournot oligopoly, Trinity Economic Paper 2005/6.

[30] Topkis, D. M., 1998. Supermodularity and complementarity, Princeton University Press, Princeton, N.J.

[31] Vega-Redondo, F., 1997. The evolution of Walrasian behavior, Econometrica 65, 375-384.

[32] Vives, X., 2000. Oligopoly pricing: old ideas and new tools, The MIT Press, Cambridge, M.A.

[33] Young, H. P., 1993. The evolution of conventions, Econometrica 61, 57-84. 